Dhaka Univ. J. Sci. 65(1) 21-25, 2017 (January)

\title{
Skin Friction and Heat Transfer in Combined Convection from a Sphere to a Non-Newtonian Fluid
}

\author{
Nepal C. Roy ${ }^{1 *}$ and Amir Husen ${ }^{2}$ \\ ${ }^{1}$ Department of Mathematics, Dhaka University, Dhaka-1000, Bangladesh \\ ${ }^{2}$ Department of Business Administration, Sonargaon University, Green Road, Dhaka-1215, Bangladesh
}

(Received: 11 January 2016; Accepted: 3 August 2016)

\begin{abstract}
Steady-state laminar combined convection flow of an electrically conducting, non-Newtonian fluid past a sphere is studied numerically. The effects of the power-law index, the mixed convection parameter, the magnetic field parameter and the heat generation or absorption parameter on the skin friction and the heat transfer are illustrated. The skin friction for dilatant fluids is smaller than that for pseudoplastics. Near the stagnation point, the Nusselt number reduces rapidly for pseudoplastics and reaches sharply a maximum value for dilatant fluids. Both the skin friction and the Nusselt number decrease owing to an increase of the magnetic field parameter. Moreover the heat generation produces higher skin friction and lower Nusselt number.
\end{abstract}

Keywords: Non-Newtonian fluid; Magnetic field; Boundary layer; Heat generation

$\begin{aligned} & \text { Nomenclature } \\ & B_{0} \text { Strength of magnetic field } \\ & C_{f} \text { Skin friction } \\ & c_{p} \text { Heat capacity of the fluid } \\ & F \text { Dimensionless stream function } \\ & g \text { acceleration due to gravity } \\ & k_{c} \text { Thermal conductivity } \\ & K \text { Fluid consistency index for power-law } \\ & \text { fluid } \\ & n \text { Index of power-law viscosity model } \\ & N u \text { Nusselt number } \\ & Q_{0} \text { Heat generation } \\ & \text { coefficient } \\ & r \text { Distance from a point on the surface to } \\ & \text { the axis of symmetry } \\ & R \text { Radius of the sphere } \\ & T \text { Temperature } \\ & u, v \text { velocity components in } x \text { - and } y \text { - } \\ & \text { directions respectively } \\ & x \text { Arc length measured along the surface }\end{aligned}$

\section{Introduction}

Combined free and forced convection of non-Newtonian fluid has been the focus of research due to its applications in industries such as processing of molten plastics, polymers, food stuff or slurries. The forced convection heat transfer between a horizontal cylinder and a power-law fluid of infinitely large Prandtl numbers was first studied by Acrivos et al. ${ }^{1}$. Kim et al. ${ }^{2}$ theoretically examined the momentum and heat transfer in power-law fluid flow over arbitrarily shaped two-dimensional or axisymmetrical bodies. On the other hand, Nakayama and Koyama ${ }^{3}$ utilized the von Karman integral method to obtain an asymptotic solution for forced convection in high non-Newtonian Prandtl number fluids. Mucoglu and Chen ${ }^{4}$ analyzed the combined forced and free convection across a horizontal cylinder. In addition the authors studied the flow- and heat-transfer characteristics of laminar mixed forced and free convection about a sphere with isothermal surface ${ }^{5}$ and constant heatflux surface ${ }^{6}$. Lien and Chen ${ }^{7,8}$ examined the forced and mixed convection flow of micropolar fluid about a permeable sphere with various prescribed thermal conditions on the surface. It should be mentioned that most

\author{
of the sphere \\ $y$ Distance normal to the surface \\ $X, Y$ Dimensionless variables \\ Greek Symbols \\ $\alpha$ Thermal diffusivity \\ $\beta$ Coefficient of volumetric expansion \\ $\theta$ Dimensionless temperature \\ $\mu \quad$ Viscosity of the fluid \\ $\rho$ Density of the fluid \\ $\sigma_{c} \quad$ Electrical conductivity \\ $\psi$ Stream function \\ Subscripts \\ $e$ Boundary layer edge condition \\ $w$ Wall condition \\ $\infty$ Ambient condition \\ Superscripts \\ ' Derivative with respect to $Y$
}

of the works on this topic have focused particularly on the classical Newtonian fluids. But the boundary layer characteristics of mixed convection flow about a sphere for the non-Newtonian fluids with microstructures have attracted less attention of the researchers.

Now the effect of the applied magnetic field is found to be significant in many industrial applications such as electric power generation, astrophysical flows, solar power technology, space vehicle re-entry, nuclear engineering applications, etc. Sathyakrishna et al. ${ }^{9}$ examined the unsteady laminar incompressible magneto-hydrodynamic (MHD) boundary layer flow and heat transfer for an electrically conducting fluid over two-dimensional and axisymmetric bodies in the presence of an applied magnetic field. Ibrahim et al. ${ }^{10}$ focused on the unsteady MHD free convection flow past a semi-infinite vertical permeable moving plate with heat source and chemical reaction. Recently, Roy et al. ${ }^{11}$ investigated the unsteady free convection from a heated sphere in the presence of internal heat generation or absorption. 
In this paper, an analysis is presented on the laminar combined convection flow of an electrically conducting, non-Newtonian fluid about a sphere. The system of dimensionless governing equations has been solved numerically. Results are discussed in terms of the skin friction and the heat transfer due to variation of the powerlaw index, the mixed convection parameter, the magnetic field parameter and the heat generation or absorption parameter.

\section{Formulation of the Model}

Let us consider the unsteady, laminar, incompressible boundary layer flow of a micropolar fluid around a sphere of radius $R$ placed in a flow field with free stream velocity $u_{\infty}$. Figure 1 illustrates the flow pattern and coordinate system. We assume that $x$ is the arc length measured along the surface of the sphere from the lower stagnation point and $y$ is the distance normal to the surface. The surface of the sphere is maintained at a uniform temperature $T_{w}$ while $T_{\infty}$ is the temperature of the ambient fluid. Also the convective forced flow is considered to be acted upon the fluid in the upward direction. However, the gravitational force is in the downward direction. It is noted that the buoyancy force resulting from the temperature difference $T_{w}>T_{\infty}$ assists the forced flow. In the Boussinesq approximation, that is, where variable fluid properties are ignored except that the density variations within the fluid causes the buoyancy forces, the equations of motion are

$$
\begin{aligned}
& \frac{\partial}{\partial x}(r u)+\frac{\partial}{\partial y}(r v)=0, \\
& u \frac{\partial u}{\partial x}+v \frac{\partial u}{\partial y}=u_{e} \frac{\partial u_{e}}{\partial x}+\frac{K}{\rho} \frac{\partial}{\partial y}\left(\left|\frac{\partial u}{\partial y}\right|^{n-1} \frac{\partial u}{\partial y}\right) \\
& +g \beta_{T}\left(T-T_{\infty}\right) \sin \left(\frac{x}{R}\right)-\frac{\sigma_{c} B_{0}^{2}}{\rho} u \\
& u \frac{\partial T}{\partial x}+v \frac{\partial T}{\partial y}=\alpha \frac{\partial^{2} T}{\partial y^{2}}+\frac{Q_{0}}{\rho c_{p}}\left(T-T_{\infty}\right) .
\end{aligned}
$$

where $u$ and $v$ are the velocity components in the $x$ - and $y$ directions, respectively, $K$ is the fluid consistency index for power-law fluid, $\rho$ is the density of the fluid, $g$ is the acceleration due to gravity, $\beta$ is the coefficient of volumetric expansion, $\alpha$ is the thermal diffusivity, $c_{p}$ is the heat capacity of the fluid, $T$ is the temperature of the fluid in the boundary layer, $\sigma_{c}$ is the electrical conductivity and $B_{0}$ is the strength of magnetic field which is assumed to be applied in the $y$ direction, normal to the surface. Moreover, $Q_{0}$ is the heat generation $(>0)$ or absorption $(<0)$ coefficient and $Q_{0}\left(T-T_{\infty}\right)$ is the volumetric rate of heat generation or absorption from a source or sink of heat within the fluid.

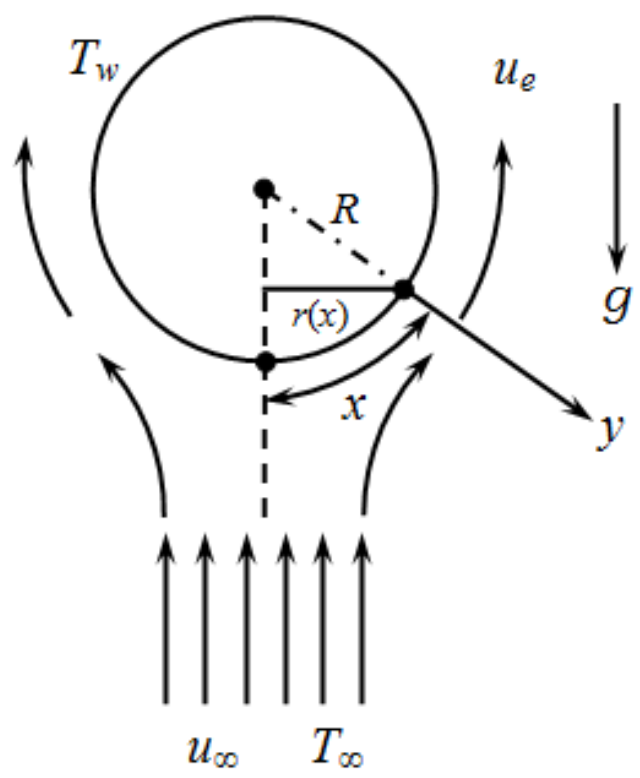

Fig. 1. Flow pattern and coordinate system

The appropriate boundary conditions are

$u=v=0, T=T_{w} \quad$ at $y=0$

$u=u_{e}, v=0, T=T_{\infty}$ as $y \rightarrow \infty$.

The radial distance $r(x)=R \sin (x / R)$ and the free stream velocity $u_{e}(x)$ is assumed to be of the form

$u_{e}(x)=\frac{3}{2} u_{\infty} \sin \left(\frac{x}{R}\right)$.

Introducing the following group of transformations

$$
\begin{aligned}
& X=\frac{x}{R}, Y=\left(\frac{\operatorname{Re}}{X}\right)^{\frac{1}{n+1}} U_{e}^{\frac{2-n}{n+1}}\left(\frac{y}{R}\right), \\
& U_{e}=\frac{u_{e}}{u_{\infty}}, \theta(X, Y)=\frac{T-T_{\infty}}{T_{w}-T_{\infty}}, \\
& \psi=r R u_{\infty}\left(\frac{X}{\operatorname{Re}}\right)^{\frac{1}{n+1}} U_{e}^{\frac{2 n-1}{n+1}} F(X, Y),
\end{aligned}
$$

and defining the stream function, $\psi$, as

$u=\frac{1}{r} \frac{\partial \psi}{\partial y}$ and $v=-\frac{1}{r} \frac{\partial \psi}{\partial x}$,

we obtain from (2)-(3):

$$
\begin{aligned}
& \left(\left|F^{\prime \prime}\right|^{n-1} F^{\prime \prime}\right)^{\prime}+A(X) F F^{\prime \prime}+B(X)\left(1-F^{2}\right) \\
& +\operatorname{Ri} \theta \frac{X \sin X}{U_{e}^{2}}-M \frac{X}{U_{e}} F^{\prime} \\
& =X\left(F^{\prime} \frac{\partial F^{\prime}}{\partial X}-F^{\prime \prime} \frac{\partial F}{\partial X}\right)
\end{aligned}
$$




$$
\begin{aligned}
& \frac{E(X)}{\operatorname{Pr}} \theta^{\prime \prime}+A(X) F \theta^{\prime}+\frac{\Omega X}{U_{e}} \theta \\
& =X\left(F^{\prime} \frac{\partial \theta}{\partial X}-\theta^{\prime} \frac{\partial F}{\partial X}\right)
\end{aligned}
$$

where

$$
\begin{aligned}
& B(X)=\frac{X}{U_{e}} \frac{d U_{e}}{d X} \\
& A(X)=\frac{1}{n+1}+\frac{2 n-1}{n+1} B(X)+\frac{X}{r} \frac{d r}{d X} \\
& E(X)=U_{e}^{3(1-n) /(n+1)} X^{(n-1) /(n+1)}
\end{aligned}
$$

In the above equations, $R i=G r / \operatorname{Re}^{2 /(2-n)}$ is the mixed convection parameter, $G r=(K / \rho)^{2 /(n-2)} \quad g \beta_{T}\left(T_{w} \quad-\right.$ $\left.T_{\infty}\right) R^{(2+n) /(2-n)}$ is the Grashof number, $\operatorname{Re}=\rho u_{\infty}^{2-n} R^{n} / K$ is the Reynolds number, $M=\sigma_{c} B_{0}^{2} R /\left(\rho u_{\infty}\right)$ is the magnetic field parameter, $\operatorname{Pr}=\left(u_{\infty} R / \alpha\right) \operatorname{Re}^{-2 /(n+1)}$ is the Prandtl number and $\Omega=Q_{0} R /\left(\rho c_{p} u_{\infty}\right)$ is the heat generation (>0) or absorption $(<0)$ parameter.

The associated boundary conditions are

$$
\begin{aligned}
& F=F^{\prime}=0, \theta=1 \text { at } Y=0 \\
& F^{\prime}=1, \theta=0 \text { as } Y \rightarrow \infty .
\end{aligned}
$$

Of interest are the skin friction and the Nusselt number which are defined, respectively, by

$$
\begin{aligned}
& C_{f}=\frac{1}{\rho u_{\infty}^{2}}\left[\mu \frac{\partial u}{\partial y}\right]_{y=0}, \\
& N u=-\frac{k_{c} R}{k_{c}\left(T_{w}-T_{\infty}\right)}\left(\frac{\partial T}{\partial y}\right)_{y=0} .
\end{aligned}
$$

Using the relations (7) into (14)-(15), we find

$$
\begin{aligned}
& \operatorname{Re}^{1 /(n+1)} C_{f}=X^{n /(n+1)} U_{e}^{3 n /(n+1)}\left[F^{\prime \prime}(X, 0)\right]^{n} \\
& \operatorname{Re}^{-1 /(n+1)} N u=X^{-1 /(n+1)} U_{e}^{(2-n) /(n+1)} \theta^{\prime}(X, 0)
\end{aligned}
$$

The system of equations (9) and (10) subject to the boundary conditions (12) and (13) are solved using straight forward finite difference method $^{12}$. In this method, equations (9) and (10) are reduced to a system of second order differential equations taking $U=f^{\prime}$ and $V=f$ and then they are discretized using central difference approximation along $Y$-direction while forward difference is used in $X$-direction. Thus we have a system of tri-diagonal algebraic equations of the form:

$$
A_{k} W_{i-1, j}+B_{k} W_{i, j}+C_{k} W_{i+1, j}=D_{k} \text {. }
$$

In the above equations, the subscript $k(=1,2)$ represents the functions $U$ and $\theta, i(=1,2,3, \ldots, M)$ and $j(=1,2,3, \ldots, N)$ correspond to the grid points in $Y$ and $X$ directions respectively. The $A_{k}, B_{k}, C_{k}$ and $D_{k}$ can be obtained easily utilizing the discretization techniques mentioned above. It is worthy of mention that for fixed values of $j$, the tri-diagonal equations (18) for $i(=1,2,3, \ldots, M)$ are solved using the well-known Thomas Algorithm [13]. The computation is then performed in $j$ direction. The convergence criteria for the steady state solutions are chosen in such a way that the difference between the values of the function $f(X, Y)$ in two consecutive iterations is less than $10^{-6}$. The computational domain $(X, Y)$ is discretized taking the step sizes $\Delta X$ and $\Delta Y$ respectively. After doing the grid independency test, the final mesh sizes are chosen to be $\Delta X=0.0005$ and $\Delta Y=$ 0.01 so that the solutions are grid independent.

Having obtained the values of $F^{\prime \prime}(X, 0)$ and $\theta^{\prime}(X, 0)$, the behavior of the skin friction and the heat transfer is illustrated using the relations (16) and (17).

\section{Results and Discussion}

Results are discussed in terms of the influences of the physical parameters, namely, the power-law index, $n$, the mixed convection parameter, $R i$, the magnetic field parameter, $M$ and the heat generation or absorption parameter, $\Omega$. We consider $\operatorname{Pr}=100.0, R i=2.0, M=0.5$ and $\Omega=-0.5$ except the variation of a parameter shown in each figure.

The effects of the power-law index, $n$, on the skin friction and the Nusselt number are shown in Figures 2 and 3, respectively. It is found that the dilatant fluids $(n>1.0)$ exhibit lower and pseudoplastics $(n<1.0)$ generate higher skin friction than Newtonian fluids $(n=1.0)$. On the other hand, the Nusselt number relating to Newtonian fluids $(n=$ $1.0)$ decreases monotonically along the curved surfaces. For Pseudoplastics $(n<1.0)$, the Nusselt number increases rapidly near the stagnation point $(X=0.0)$. Then it gradually increases and gets a maximum value. For further increase of the angular distance, the Nusselt number shows the general trend as like as the Newtonian fluids. However, the Nusselt number for dilatant fluids $(n>1.0)$ decreases drastically in the vicinity of $X=0$ and then it decreases almost linearly.

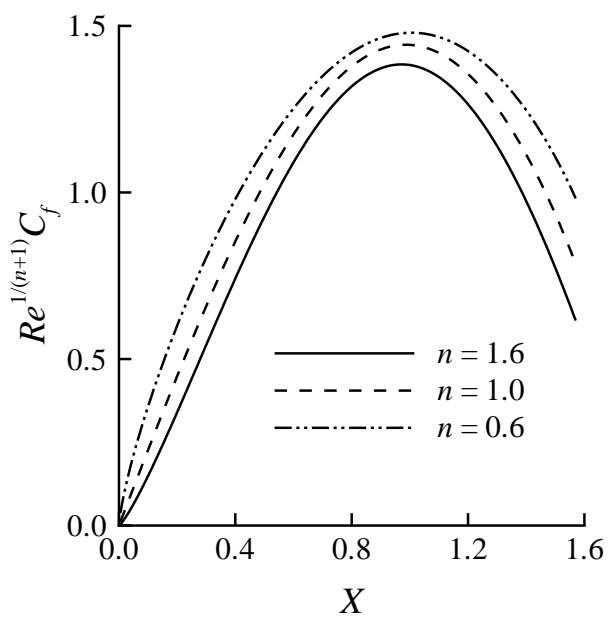

Fig. 2. Angular distribution of skin friction for different values of $n$. 


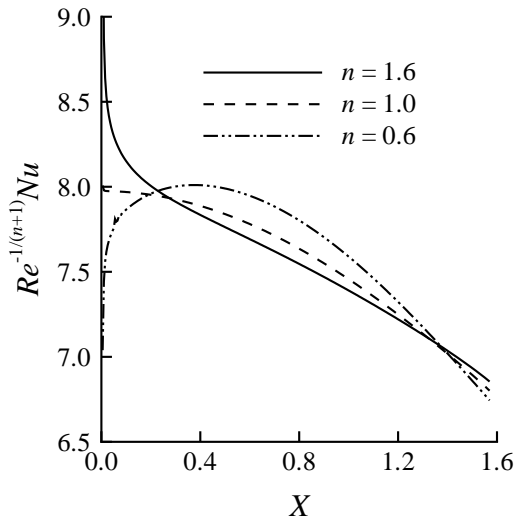

Fig. 3. Angular distribution of Nusselt number for different values of $n$.

Figures 4 and 5 illustrate the variation of the skin friction and the Nusselt number with the mixed convection parameter, Ri. It is observed that the skin friction and the Nusselt number for both pseudoplastics and dilatant fluids increase due to increase of the mixed convection parameter, $R i$. The reason for such a behavior is that the forced convection is enhanced by increasing the buoyancy force. Results also indicate that the skin friction is significantly affected by the mixed convection parameter while the influence on the Nusselt number is rather weak.

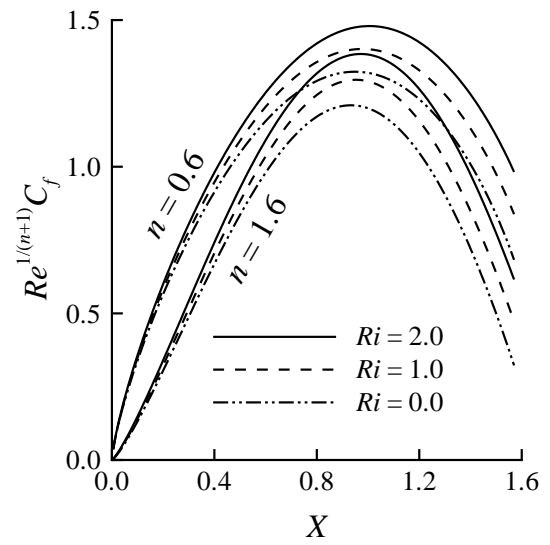

Fig. 4. Angular distribution of skin friction for different values of $R i$.

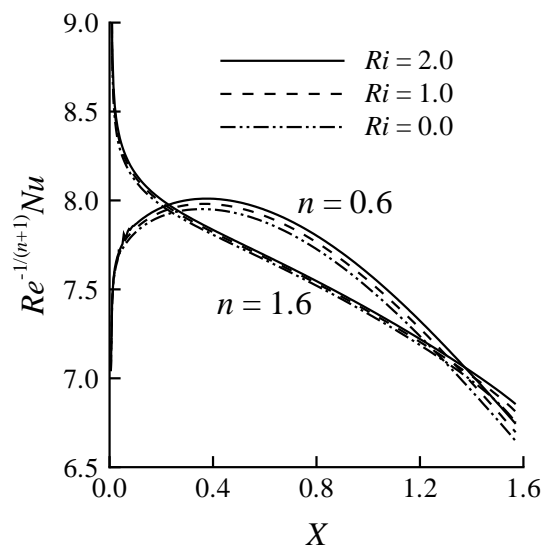

Fig. 5. Angular distribution of Nusselt number for different values of $R i$.

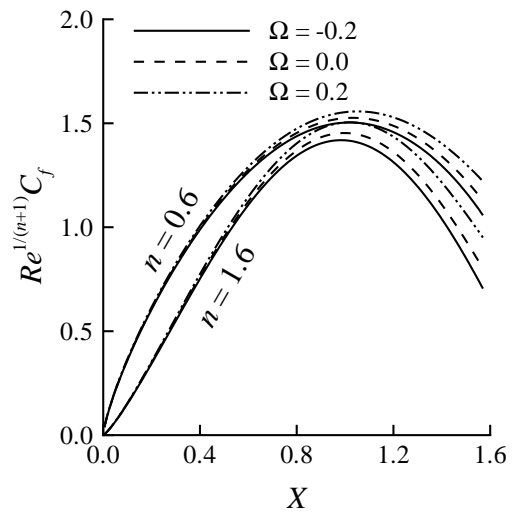

Fig. 6. Angular distribution of skin friction for different values of $\Omega$.

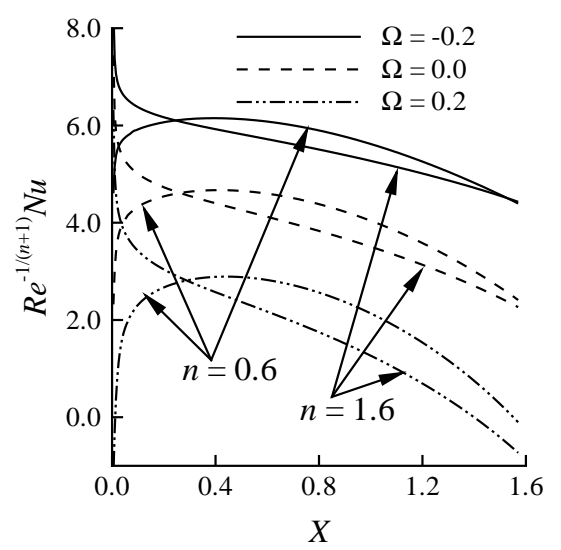

Fig. 7. Angular distribution of Nusselt number for different values of $\Omega$.

The effects of the heat generation $(\Omega>0)$ or absorption $(\Omega<0)$ on the skin friction and the Nusselt number are depicted in Figures 6 and 7, respectively. It is found that the friction factor increases with an increase of heat generation and a decrease of heat absorption. But the Nusselt number decreases due to an increase of heat generation and a decrease of heat absorption. The influence of $\Omega$ on the skin friction and the Nusselt number is appreciable.

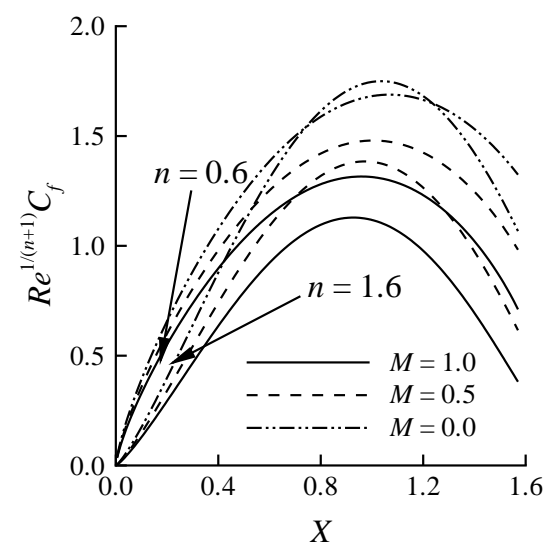

Fig. 8. Angular distribution of skin friction for different values of $M$. 


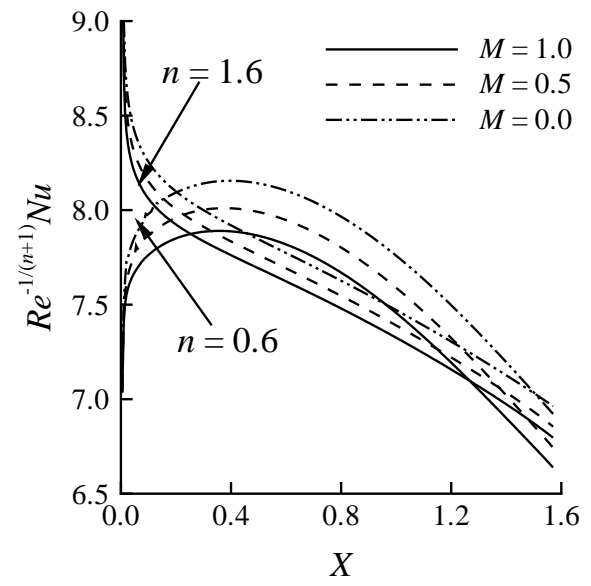

Fig. 9. Angular distribution of Nusselt number for different values of $M$.

Figures 8 and 9 demonstrate the effects of the magnetic field parameter, $M$, on the skin friction and the Nusselt number. Results indicate that the skin friction and the Nusselt number considerably decrease owing to an increase of $M$. The reason for such a reduction is that the applied magnetic field delays the fluid flow and consequently reduces the skin friction and the Nusselt number.

\section{Conclusions}

We study the steady, laminar, mixed convection boundary layer flow of an electrically conducting non-Newtonian fluid past a sphere. In comparison with Newtonian fluids, the dilatant fluids generate lower and pseudoplastics produce higher skin friction. The Nusselt number is completely different for non-Newtonian fluids from the Newtonian fluid. Near the stagnation point, the Nusselt number gets a peak value for pseudoplastics while it decreases rapidly for dilatant fluids. The skin friction and the Nusselt number significantly reduce with increasing values of magnetic field parameter. Moreover, the heat generation enhances the skin friction and impedes the Nusselt number.

\section{References}

1. Acrivos, A., M. J. Shah, and E. E. Peterson, 1965. On the solution of the two-dimension boundary-layer flow equations for a non-Newtonian power law fluid. Chem. Eng. Sci., 20, 101-105.
2. Kim, H. W., D. R. Jeng, and K. J. DeWitt, 1983. Momentum and heat transfer in power-law fluid flow over twodimensional or axisymmetrical bodies. Int. J. Heat Mass Transfer, 26, 245-259.

3. Nakayama, A. and H. Koyama, 1986. An asymptotic expression for forced convection in non-Newtonian powerlaw fluids. Int. J. Heat Fluid Flow, 3, 99-101.

4. Mucoglu, A. and T. S. Chen, 1977. Analysis of combined forced and free convection across a horizontal cylinder. Can. J. Chem. Eng., 55, 265-271.

5. Mucoglu, A. and T. S. Chen, 1977. Analysis of mixed forced and free convection about a sphere, Int. J. Heat Mass Transf., 20, 867-875.

6. Mucoglu, A. and T. S. Chen, 1978. Mixed convection about a sphere with uniform surface heat flux. J. Heat Transfer, 100(3), 542-544.

7. Lien, F.-S. and C.-C. Chen, 1986. Analysis of forced convection micropolar boundary layer about a permeable sphere. Int. J. Eng. Sci., 24, 991-999.

8. Lien, F.-S. and C.-C. Chen, 1987. Mixed convection of micropolar fluid about a sphere with blowing and suction. Int. J. Eng. Sci., 25, 775-784.

9. Satyakrishna, M., S. Roy, and G. Nath, 2001. Unsteady twodimensional and axisymmetric MHD boundary-layer flows. Acta Mechanica, 150, 67-77.

10. Ibrahim, F. S., A. M. Elaiw, and A. A. Bakr, 2008. Effect of the chemical reaction and radiation absorption on the unsteady MHD free convection flow past a semi infinite vertical permeable moving plate with heat source and suction. Commun. Nonlin. Sci. Numer. Simulat., 13, 1056-1066.

11. Roy, N. C., M. A. Hossain, and R. S. R. Gorla, 2015. Unsteady free convection from a heated sphere in the presence of internal heat generation or absorption, Int. J. Therm. Sci., 98, 237-244.

12. Hossain, M. M., A. C. Mandal, N. C. Roy, and M. Hossain, A., 2013. Fluctuating flow of thermomicropolar fluid past a vertical surface. Applications and Applied Mathematics: An International Journal, 8, 128-150.

13. Blottner, F. G., 1970. Finite-difference methods of solution of the boundary layer equations, AIAA Journal, 8(2), 193-205. 
